\title{
Production and characterization of low molecular weight heparin obtained by modified double emulsion method with solvent evaporation
}

\begin{abstract}
The main anticoagulant agents currently used in the treatment of coagulation disorders are low molecular weight heparin (LMWH) and unfractionated heparin (UFH) LMWH is formed by smaller UFH chains obtained by multiple chemical or enzymatic depolymerization processes and has advantages over UFH in that it does not cause severe toxicity and has more predictable pharmacokinetic properties. The use of LMWH, even with its advantages, is still limited due to the need for daily subcutaneous injections because it is of animal origin and does not have a definite chemical composition. These factors motivated the researchers to explore new delivery systems of this medication for better therapeutic results. The objective of this study was to produce and characterize LMWH nanoparticles through the solvent-evaporated double emulsion method using poly lacticco-glycolicacid (PLGA) and polyvinyl alcohol (PVA), calculate encapsulation efficiency (EE) and evaluate stability for future in vitro and in vivo testing. The methodology used was effective and produced LMWH nanoparticles of medium diameter $224.8 \pm 0.85$, zeta potential $-17.27 \pm 1.10$ and polydispersity index $0.07 \pm 0.05$. Despite the hydrophilic characteristic of LMWH, the obtained EE was $66.5 \%$, a very promising result that suggests the effectiveness of the nanoencapsulation method used resulting in an alternative for the development of new anticoagulant therapies.
\end{abstract}

Keywords: low molecular weight heparin, anticoagulants, nanoparticles
Volume 7 Issue 6 - 2019

\author{
Roberta Carolina Rangel de Oliveira, Tamara \\ Gonçalves de Araújo \\ Postgraduate Program in Technological Development and \\ Innovation in Medicines (PpgDITM). Department of Pharmacy, \\ Federal University of Ceará, Brazil
}

\begin{abstract}
Correspondence: Roberta Carolina Rangel de Oliveira, Faculdade de Farmácia, Odontologia e Enfermagem-FFOE, Rua Capitão Francisco Pedro, I 210 -Rodolfo Teófilo CEP 60.430-I70 Fortaleza, Ceará, Brasil,Tel +55 85988233572 , Email rocarolina@hotmail.com
\end{abstract}

Received: September 28, 2019 | Published: November 08, 2019
Abbreviations: LMWH, low molecular weight heparin; UFH, unfractionated heparin; PLGA, lactic-co-glycolic acid; PVA, polyvinyl alcohol; EE, encapsulation efficiency

\section{Introduction}

Currently, thromboembolic diseases are responsible for a significant portion of morbidity and mortality. ${ }^{1}$ The drugs of choice for coagulation disorders are unfractionated heparin (UFH) and low molecular weight heparin (LMWH). UFH has had problems with its use as an unpredictable effect requiring monitoring, risk of bleeding and thrombocytopenia because it acts directly on fibrin formation. Besides that, this drug can cause osteoporosis by binding to osteoblasts, and due to its animal origin may present a risk of contamination by pathogens. ${ }^{2}$ Studies state that LMWH has advantages over UFH, as a more predictable response, lower risk of bleeding and thrombocytopenia, and is the most suitable anticoagulant for pulmonary embolism and deep vein thrombosis. ${ }^{2}$ However, it is known that the use of this heparin still has complications due to the need for daily subcutaneous injections, parenteral administration route, high molecular weight and anionic character. ${ }^{3}$

As the pharmacokinetic properties of LMWH are more predictable, there was a need to work with new drug delivery systems to achieve better therapeutic results, better bioavailability and fewer side effects. ${ }^{4}$
Nanocapsulation of a drug may be a good alternative for improving its pharmacokinetic profile and efficacy, as well as reducing side effects and increasing its stability. ${ }^{5}$ Studies have reported promising results in the development of micro and nanoparticles using LMWH1-coloaded polylactic glycolic acid (PLGA). PLGA is widely used because it is biodegradable, biocompatible and versatile and has already been approved by the European Food and Drug Administration as an excipient for parenteral products. ${ }^{6}$ Thus, the purpose of this study was the LMWH nanoencapsulation and characterization of nanoparticles obtained from the solvent-evaporated double emulsion method, using PLGA and polyvinyl alcohol (PVA) for future in vitro and in vivo efficacy tests.

\section{Methodology}

Nanoparticle Development: Commercially purchased aqueous LMWH solution (Clexane $100 \mathrm{mg} / \mathrm{ml}$ ) was dissolved in $5 \mathrm{ml}$ diclomethane: PLGA (50:50) solution and sonicated (Sonifier ${ }^{\circledR}$ Model W-450D Branson) at 50\% amplitude for 15 seconds in an ice bath forming the first emulsion. Immediately thereafter, a 1.0\% PVA solution was added and sonication again forming the double emulsion. The suspension was allowed to stir for 6 hours to fully evaporate the solvent (dichloromethane). Subsequently, the suspension was added to a separating filter (Amicon Ultra-15ml) and centrifuged for $15 \mathrm{~min}$ at $3000 \mathrm{rpm}$ (Figure 1). ${ }^{6}$ 


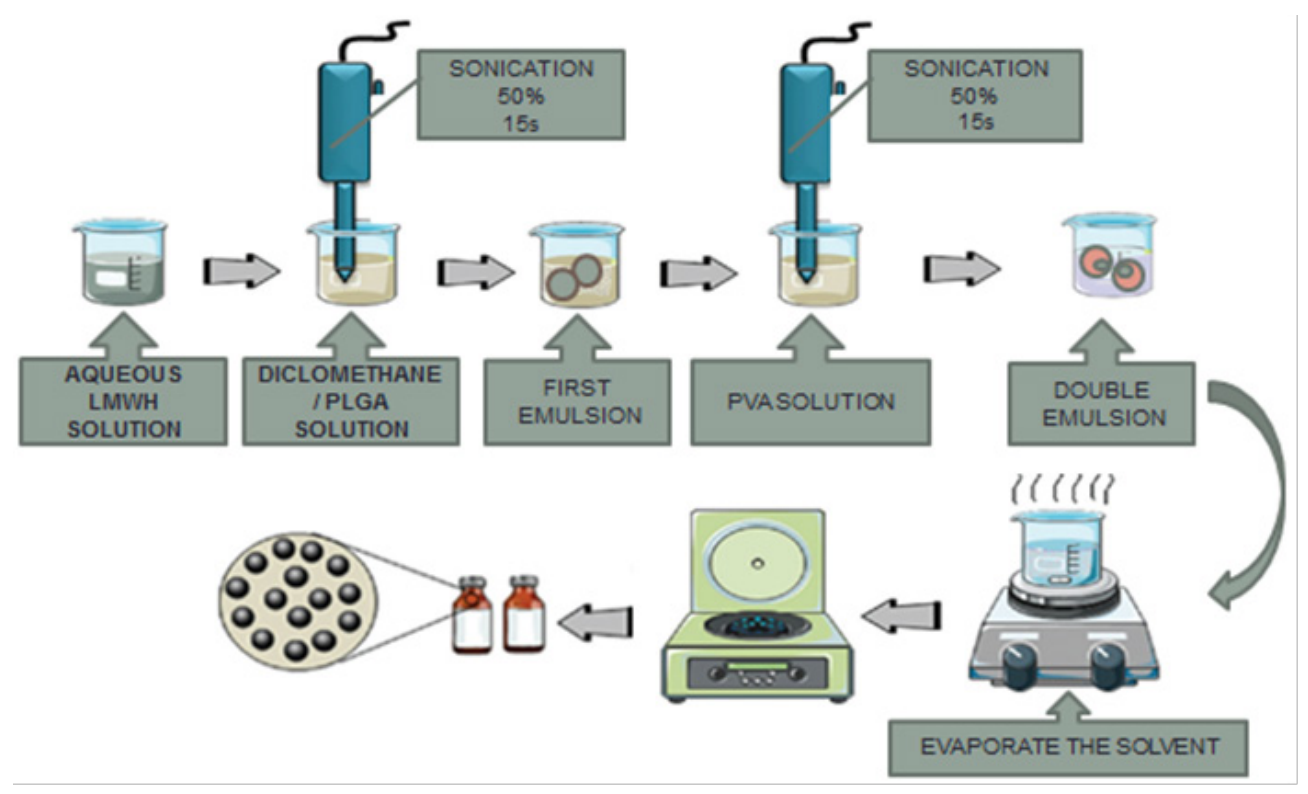

Figure I Nanoparticle development.

Characterization: Nanoparticles were characterized by surface morphology, surface coating, particle size, polydispersity index, zeta potential, drug loading and encapsulation efficiency by photon correlation spectroscopy (PCS) by Zeta Sizer Malvern (Malvern Instruments Corp). Measurements were made in KCL solution $(1 \mathrm{mM})$. Stability was measured at times 0 and 30 days; For this, the dispersions were kept at room temperature and protected from light and heat for 30 days. $^{3}$

Nanoecapsulation efficiency tests: Nanoparticulate encapsulated LMWH was quantified by turbidimetric method. To perform the calibration curve a stock solution of $10 \mathrm{mg} / \mathrm{ml}$ LMWH was prepared. From this solution successive dilutions were made to obtain the concentrations: $4 \mathrm{mg} / \mathrm{ml}, 3 \mathrm{mg} / \mathrm{ml}, 2 \mathrm{mg} / \mathrm{ml}$, and $1 \mathrm{mg} /$ $\mathrm{ml}$. The absorbances of these solutions were read on a UVVis spectrophotometer (Thermo Scientific Genesis 10S UV-Vis Spectrophotometer) at a wavelength of $290 \mathrm{~nm}$ in triplicate. ${ }^{8}$ Then the supernatant was measured at a wavelength of $290 \mathrm{~nm}$. LMWH encapsulation efficiency (EE) was calculated by the following equation:

$$
E E(\%)=\frac{[\text { Intial drug total }]-[\text { Drug in the sup erna } \tan t]}{[\text { Intial drug total }]} \times 100
$$

\section{Results and discussion}

\section{Nanoencapsulations}

Homogeneous and stable LMWH nanoparticles were obtained with $217.9 \pm 2.5 \mathrm{~nm}$ of particle diameter $(\mathrm{Dm})$, zeta potential $(\mathrm{PZ})$ $-17.06 \pm 0.51 \mathrm{mV}$ and polydispersion index (IPD) of $0.067 \pm 0.05$. Surface chemistry critically affects the way nanoparticles interact with each other and with the environment, and even more with cells. ${ }^{5}$ Nanoparticles prepared with PLGA have invariably negative charge. ${ }^{9}$ The prepared samples presented satisfactory Dm of particles for nanostructured systems with low standard deviation, indicating robustness in the preparation. In addition, the low polydispersion value (IPD) obtained revealed the homogeneity of the samples.
Similar results were obtained by Chereddy KK and colleagues, Dm 163.0 2.2, PZ $-19.2 \pm 2.5 \mathrm{mV}$ and IPD $0.15 \pm 0.05$, when nanoencapsulating a healing peptide using a similar methodology. ${ }^{6}$ In the article by Prado LB et al., LMWH was nanoencapsulated using poly (E- caprolactone) (PCL) and chitosan and the results obtained were Dm $512.5 \mathrm{~nm}$, IPD 0.409 and PZ $+30.9 \mathrm{mV}$ of chitosan in the process. In this study the solvent-evaporated double emulsion method produced nanoparticles larger than $400 \mathrm{~nm}$ and IPD greater than 0.2 characterizing system polydispersion, in other words, nanoparticles of varying sizes, ${ }^{7}$ suggesting that the efficiency of developing a nanocarrier system is related to the methodology employed and the selected inputs.

\section{Stability}

After preparation, the nanoparticles resulted in formulations with homogeneous macroscopic appearance, milky, white and opalescent appearance. The suspensions showed bluish reflection, resulting from the Brownian motion of the suspended nanoparticles. The visual appearance of LMWH nanoparticle suspensions was similar to that described in the literature and remained homogeneous over the stability study time. The results are described in Table 1 .

Table I Nanoparticle stability

\begin{tabular}{llll}
\hline Time & $\begin{array}{l}\text { Particle } \\
\text { diameter } \\
(\mathbf{n m})\end{array}$ & $\begin{array}{l}\text { Zeta } \\
\text { potential } \\
(\mathbf{m V})\end{array}$ & $\begin{array}{l}\text { Polydispersion } \\
\text { index (IPD) }\end{array}$ \\
\hline 0 day & $217,9 \pm 2,5$ & $-17,06 \pm 0,5 \mid$ & $0,067 \pm 0,03$ \\
30 days & $228,4 \pm 3,2$ & $-17,3 \pm 0,91$ & $0,07 \pm 0,05$ \\
\hline
\end{tabular}

\section{Encapsulation efficiency}

As LMWH is hydrophilic, the literature reports that the encapsulation of this type of substance is complex, since there is a greater diffusion of the active to the external aqueous phase before the precipitation of the polymer, thus reducing the encapsulation efficiency. ${ }^{7}$ In this study, the LMWH Encapsulation Efficiency was $66.5 \%$, showing that despite the hydrophilic characteristics of the 
drug, the methodology employed in the development of the carrier system was satisfactory, a result similar to that obtained by Jogala et al, 2015 which obtained 46-70\% EE, when nanoencapsular LMWH using PVA and PLGA9 double emulsion methodology. Thus, it can be stated that the EE obtained with the described methodology agrees with the literature and, therefore, satisfactory.

\section{Conclusion}

The present study confirmed that even though LMWH is hydrophilic, and it can be nanoencapsulated by solvent-evaporated double emulsion method using Poly (lactic-co- glycolic acid) (PLGA) and Polyvinyl Alcohol (PVA). The encapsulation efficiency obtained was satisfactory when compared to others described in the literature. The nanoparticles obtained may represent an interesting and viable alternative for the treatment of thromboembolic disorders.

\section{Acknowledgments}

The authors would like to thank the Brazilian Higher-Level Personnel Improvement Coordination Agency (CAPES) for their financial support.

\section{Conflicts of interest}

Authors declare that there is no conflict of interest.

\section{References}

1. Akhtar F, Wan X, Wu G, et al. Low-Molecular-Weight Heparins: Reduced Size Particulate Systems for Improved Therapeutic Outcomes. Molecules. 2018;23(7):1757.
2. De Oliveira Barddal HP, Gracher AHP, Simas-Tosin FF, et al. Anticoagulant activity of native and partially degraded glycoglucuronomannan after chemical sulfation. Int J Biol Macromol. 2015;80:328-333.

3. Al-Hilal TA, Park J, Alam F, et al. Oligomeric bile acid-mediated oral delivery of low molecular weight heparin. $J$ Control Release. 2014;175:17-24

4. Ibrahim SS, Osman R, Awad GA, et al. Low molecular weight heparins for current and future uses: approaches for micro-and nano-particulate delivery. Drug Deliv. 2016;23(8):2661-2667.

5. Da Silva LC, Garcia T, Mori M, et al. Preparation and characterization of polysaccharide-based nanoparticles with anticoagulant activity. Int $J$ Nanomedicine. 2012;7:2975-2986.

6. Chereddy KK, Her CH, Comune M, et al. PLGA nanoparticles loaded with host defense peptide LL37 promote wound healing. J Control Release. 2014;194:138-147.

7. Prado LB, Huber SC, Barnabé A, et al. Characterization of pcl and chitosan nanoparticles as carriers of enoxaparin and its antithrombotic effect in animal models of venous thrombosis. Journal of Nanotechnology. 2017;2017:4925495.

8. Marotta-Oliveira SSA, Marchetti JM. Determination of fractionated heparin in pharmaceutical dosage forms using turbidimetry. Quim Nova. 2011;34(6):1063-1067.

9. Jogala S, Rachamalla SS, Aukunuru J. Development of subcutaneous sustained release nanoparticles encapsulating low molecular weight heparin. J Adv Pharm Technol Res. 2015;6(2):58-64. 